Original Research Paper

\title{
Development of an Immunomagnetic Separation Method for Viable Salmonella Typhimurium Detected by Flow Cytometry
}

\author{
${ }^{1,2}$ Shakil Ahmed, ${ }^{2}$ Horst-Günter Rubahn and ${ }^{1}$ Helmut Erdmann \\ ${ }^{1}$ Department of Biotechnology, Flensburg University of Applied Sciences, Kanzleistr.91-93, 24943 Flensburg, Germany \\ ${ }^{2}$ Mads Clausen Institute, Faculty of Engineering, University of Southern Denmark, Alsion 2 DK-6400 Sønderborg, Denmark
}

\author{
Article history \\ Received: 15-09-2016 \\ Revised: $15-11-2016$ \\ Accepted: 24-11-2016 \\ Corresponding Author: \\ Shakil Ahmed \\ Department of Biotechnology, \\ Flensburg University of \\ Applied Sciences, \\ Kanzleistr.91-93, 24943 \\ Flensburg, Germany \\ Email: shakil.ahmed@hs-flensburg.de
}

\begin{abstract}
A very small number of bacterial pathogens may have fatal effects on food safety. In spite of having great advancements in bioanalytical methods, most of the accepted detection methods are still cultivation based and thus time consuming. This leads to an intense need for efficient and rapid methods for detection of food-related bacteria. In this study, a flow cytometry based immunomagnetic separation (IMS) method for the isolation and enrichment of Salmonella Typhimurium from liquid samples was developed and optimized. Both polyclonal and monoclonal antibodies have been used to couple with 1 micron sized paramagnetic particles for the preparation of immunomagnetic beads (IMBs). The most suitable antibody was chosen by applying an enzyme linked immunosorbent assay (ELISA), whereas living bacteria were detected by flow cytometry. The parameters for both IMS and flow cytometry e.g., concentration of bead and bacteria, immunocapture time, staining and buffering conditions for the viability assays were optimized. The capture efficiency of IMS was $>98 \%$ for a range of Salmonella Typhimurium cell concentrations from $10^{3}$ to $10^{5} / \mathrm{mL}$ using $10^{8} / \mathrm{mL}$ bead concentration. The method proved to have high $(98 \%)$ specificity towards Salmonella Typhimurium and very low $(<5 \%)$ binding with non-target bacterial strains.
\end{abstract}

Keywords: Immunomagnetic Separation, Lab-on-a-Chip, Flow Cytometry, Salmonella Typhimurium

\section{Introduction}

Due to an increased number of recalls and cases involving microbial contamination and adulteration of food, food processing industries require on-line fast, sensitive and early detection methods for microbial contaminants to avoid withdrawing a large amount of products from the market. Both pathogenic bacteria and non-pathogenic spoilage microorganisms cause severe problems in the food industry. The most common foodborne pathogens are Salmonella spp., Escherichia coli O157:H7, Staphylococcus aureus, Campylobacter jejuni and Listeria monocytogenes, which have been detected in different food samples (McCabe-Sellers and Beattie, 2004; Mead et al., 1999; Batz et al., 2005).

Currently used analytical procedures in food industries are dependent on traditional culture based methods that include enrichment, plating on selective differential agar and then biochemical confirmation (USDA, 2004; USFDA, 2002). They are quite time consuming and have to be performed in off-site laboratories. In fact, the rapid and specific detection of contamination is one of the great challenges in food safety, because of the presence of complex subpopulations varying in viability, vitality, pathogenicity and physiological state (Hammes and Egli, 2010). Moreover in the event of a contamination the number of target pathogens may be very low and cannot be detected without additional enrichment steps. The necessity for capturing low numbers of specific microorganisms in food sample results in the need for efficient techniques like immunomagnetic separation (IMS), which offers several advantages over traditional culture enrichment processes.

Superparamagnetic particles can be coated with antibodies or other ligands against the target pathogen and form immunomagnetic beads (IMBs). The IMBs bind specifically to the target bacteria forming a bead-bacteria complex which can be separated by introducing an external magnetic field that finally concentrates the complex into a smaller volume. The ability to vary the antibodies, bead type and size makes IMS a versatile and flexible technique. Several methods of detection can be 
used to quantify and identify the captured bacteria after IMS. Immunomagnetic separation (IMS) has been applied in conjunction with conventional plating (Hara-Kudo et al., 2001), enzyme-linked immunosorbent assay (ELISA) (Cudjoe et al., 1995), polymerase chain reaction (PCR) (Fu et al., 2005; Wang et al., 2007; Yang et al., 2007) and flow cytometry (Hibi et al., 2006). Detection of microbial contaminants by conventional culture methods is time consuming and significantly underestimates the number of microorganisms whereas ELISA and PCR do not confirm the presence of viable bacteria. Therefore a combination of IMS and flow cytometric detection of viable bacteria after vital staining is a more appropriate approach. Flow cytometry provides a direct, rapid and accurate way of real-time monitoring of cell populations in the industrial product line. Different studies also showed the potential and scope of flow cytometry applications in single cell analysis in different fields (Bergquist et al., 2009; Vives-Rego et al., 2000).

Conventional state-of-the-art flow cytometry devices are bulky, expensive and complex and are therefore not suitable for implementation in the production line of the food processing industries. Lab-on-a-chip (LOC), in contrast, has a strong potential to be used in the field for on-site measurements since LOC devices are small, simple and cheap. They potentially also allow for fast and very sensitive detection. A LOC device integrates several laboratory functions onto a small platform and involves the handling of very small fluid volumes. In addition, the advantages to use LOC platforms include low sample and reagent consumption, high speed of analysis, high reproducibility etc. and therefore it has been proven to be useful for the detection of microbial contaminants (Kunstmann-Olsen, 2012).

The objective of this study was to develop a general model system for immunomagnetic separation method for microorganism in order to prepare efficient immunomagnetic beads (IMBs) to capture nearly all microbial contaminants of the sample with the aim to be implemented into an on-chip microfluidic environment. Combination of flow cytometry with IMS is a promising approach for such rapid detection of microbial contamination (Füchslin et al., 2010; Seo et al., 1998), however adulteration by live cell was not reported so far. In this study, we have established a method to detect magnetically separated viable bacteria (here Salmonella Typhimurium). Investigations were performed to identify a suitable bead and antibody. The bead concentration, bacteria concentration and bead-bacteria interaction periods were optimized for maximum capture efficiency which is about $100 \%$.

\section{Materials and Methods}

\section{Cultures}

Salmonella Typhimurium, (Leibniz Institute DSMZGerman Collections of Microorganisms and Cell
Cultures, DSM 11320) was grown in CASO broth (CASO-Bouillon, Merck KGaA, Darmstadt), E. coli K12 (DSM 498, DSMZ) and E. coli MM294 (DSM 5208, DSMZ) were grown in Nutrient broth (DSMZ medium 1) and Listeria innocua (DSM 20649) was grown with Brain Heart Infusion (Becton Dickinson, Sparks, Maryland). They all were cultivated at $37^{\circ} \mathrm{C}$ for $20 \mathrm{~h}$ before use. Bacteria were quantified by standard flow cytometry method and diluted with 0.22 micron sterile filtered PBST (PBS buffer at $\mathrm{pH} 7.4+0.01 \%$ Tween 20) to achieve final concentrations of $10^{3}$ to $10^{7} \mathrm{cells} / \mathrm{ml}$ and used immediately for experiment. Cultures were maintained at $-80^{\circ} \mathrm{C}$ in $10 \%$ glycerol. Dilutions of cultures were made in sterile PBST buffer.

\section{Paramagnetic Particles and Antibody}

Immunomagnetic beads coupled with antibodies specific for Salmonella spp. were used for capture and detection of bacteria. Three different antibodies were compared: rabbit IgG anti-Salmonella spp. biotinylated polyclonal antibody (antibodies-online $\mathrm{GmbH}$, Aachen, Germany), mouse IgG3 anti-Salmonella (immunogen: Salmonella LPS core) monoclonal antibody (ViroStat, Portland, USA) and mouse IgG2a monoclonal antibody (ViroStat, Portland, USA) specific to the ECA antigen common to all Enterobacteriaciae. Then $1 \mu \mathrm{m}$ Dynabeads ${ }^{\circledR}$ MyOne ${ }^{\mathrm{TM}}$ Streptavidin C1 (Invitrogen) with streptavidin coating covalently linked to the bead surfaces were coupled to biotinylated antibodies. Nonbiotinylated monoclonal antibodies were conjugated with biotin using a conjugation kit (ab102867, abcam, UK) and the standard conjugation protocol as suggested by the manufacturer.

\section{ELISA for Determining Suitable Antibody for Salmonella}

A standard protocol for an indirect ELISA method was applied, whereby $\sim 10^{5}$ bacteria were immobilized per well. Variable amounts $\left(10^{-4}-10 \mu \mathrm{g}\right)$ of the tested antibodies (biotinylated) were allowed to react with the fixed amount of antigen and subsequently Streptavidin Horseradish Peroxidase (Pierce $^{\mathrm{TM}}$ High Sensitivity Streptavidin-HRP, Thermo Fisher Scientific, USA) was used to detect the bound analyte (antibody of interest). PowerWave HT microplate spectrophotometer (BioTek, USA) was used to read the ELISA plates.

\section{Preparation of Immunomagnetic Beads (IMBs) and Protocol for IMS}

About $1 \mu \mathrm{m}$ Dynabeads ${ }^{\circledR}$ with a monolayer of streptavidin covalently bound to the surface were coupled with rabbit polyclonal antibody (biotinylated) of Salmonella spp. About $10^{6}$ beads were suspended in 1 $\mathrm{mL}$ PBST and $0.1 \mu \mathrm{g}$ of antibody was added and allowed to incubate at room temperature for $30 \mathrm{~min}$ with 1000 
rpm in a thermomixer. After binding, the beads were washed magnetically two times with PBST buffer using a Dynal Invitrogen bead separator and finally the beads were re-suspended with the same buffer and stored at 2$8^{\circ} \mathrm{C}$ for subsequent use in the experiments.

The coupling rate between paramagnetic bead and rabbit polyclonal antibody of Salmonella spp. was optimized. Antibody concentrations in the range 0.01-10 $\mu \mathrm{g}$ were applied per $1 \mathrm{mg}\left(\sim 10^{6}\right)$ of beads. To check the success of coupling, beads were stained with streptavidin Alexa-405 and analyzed in the flow cytometer. Streptavidin-Alexa 405 detects only the biotinylated antibody coated beads through the biotin-streptavidin interaction. About $0.1 \mu \mathrm{g}$ of antibody was sufficient for successful conjugation of antibodies to $99 \%$ of $1 \mathrm{mg}$ beads (results not shown).

$1 \mathrm{ml}$ of the sample (containing $10^{3}$ to $10^{7}$ bacteria $/ \mathrm{ml}$ ) in buffer (50 mM Hepes $+0.25 \%$ Glutaraldehyde) was mixed with coated paramagnetic beads $\left(\sim 10^{5}\right.$ to $\left.10^{8}\right)$. The mixture was incubated for $30 \mathrm{~min}$ at room temperature with shaking at a thermomixer at $1000 \mathrm{rpm}$. After incubation the sample was washed twice magnetically using a Dynal Invitrogen bead separator for $3 \mathrm{~min}$ and the washed beads were re-suspended with 1 $\mathrm{mL}$ of the same buffer and the captured bacteria were detected by flow cytometry after viability staining with the live cell marker 5(6)-carboxyfluorescein diacetate (CFDA). The capture efficiency was calculated by comparing the total number of viable bacteria present in the sample before IMS with bacterial cells left in the supernatant after IMS.

\section{Flow Cytometry Detection of Viable Bacteria before and after IMS Enrichment}

Determination of the total cell count in suspensions of pure cultures was carried out by using flow cytometry after staining with CFDA (Sigma-Aldrich, Germany). $500 \mu \mathrm{l}$ of cell suspension was incubated for $20 \mathrm{~min}$ at $37^{\circ} \mathrm{C}$ with $10 \mathrm{mM} \mathrm{CFDA} \mathrm{(in} \mathrm{Dimethylsulfoxide).} 50 \mu \mathrm{L}$ of counting beads $\left(\mathrm{BD}^{\mathrm{TM}}\right.$ liquid Counting Beads, BD Bioscience, Heidelberg) were added to the stained bacteria and mixed. The cell count was measured by applying the following formula:

$\frac{\text { Number of events in cell region }}{\text { Number of events in bead region }}$
$\times \frac{\text { Number of beads for each test }}{\text { Test volume }(\mathrm{ml})}$
$\times$ Dilution factor $=$ Cell concentration $($ cells $/ \mathrm{ml})$

Where, necessary, the samples were diluted just before the measurement with PBST so that the concentration measured by flow cytometry was always smaller than $10^{6} \mathrm{bacteria} / \mathrm{ml}$. At least 1,000 bead events were measured to assure statistical significance. To conduct viability staining after IMS, both unbound and beadbound bacteria were incubated for $20 \mathrm{~min}$ at $37^{\circ} \mathrm{C}$ with $10 \mathrm{mM}$ CFDA (in DMSO) and analyzed by flow cytometry (see below).

Experiments were carried out using a Cytomics FC 500 (Beckman Coulter) flow cytometer equipped with two lasers of 488 and $405 \mathrm{~nm}$ excitation. Parameters were expressed on a logarithmic scale with a total of about 50,000 cells measured for every sample. PMT voltages were adjusted using an unstained sample of bacteria and paramagnetic beads. Fluorescence of cells stained with CFDA was detected in the FL1 channel (525 BP, Excitation $488 \mathrm{~nm}$ ) whereas cells labeled with Alexa 405 were detected in FL2 channel (450 DF50, Excitation 405 $\mathrm{nm})$. The detection threshold was set at medium rate. Subsequent data analysis was performed through CXP analysis software (Beckman Coulter, USA).

\section{Results and Discussion}

\section{Detection of Suitable Antibody to Prepare Immunomagnetic Beads}

The efficiency of IMS mainly depends on the affinity of the antibody towards the target bacterium that is used for the preparation of IMBs. To identify the most suitable antibody an indirect ELISA test which is widely used due to its simplicity and quickness, was performed to compare the binding capacity of three different antibodies. In a preliminary experiment a series of antibody concentrations were tested to prepare a saturation curve (results not shown). Then the specific concentration (fixed amount of antibody) showing the highest response for the respective antibodies was used for the subsequent ELISA together with all controls (without HRP, antigen and antibody).

The result shown in Fig. 1 confirms that rabbit polyclonal antibodies specific for Salmonella were more effective than either of the two monoclonal antibodies. The difference in ELISA signals can be explained by the difference in antigen binding sites between polyclonal and monoclonal antibodies. The mouse monoclonal antibody $\operatorname{IgG} 2 \mathrm{a}$ anti-ECA and IgG3 anti-Salmonella recognize an enterobacterial common antigen and LPS core respectively whereas the rabbit polyclonal antibody against Salmonella recognizes many antigenic serotypes. The experimental results suggest that rabbit polyclonal antibodies of Salmonella should be used to prepare IMBs for the separation experiments.

\section{Viability Staining of Bacteria}

After IMS the bacteria underwent viability staining followed by quantification through flow cytometry. The number of bound bacteria can be determined either staining the separated bacteria bound with magnetic beads or by staining the supernatant with the bacteria 
that did not bind with beads. Since the IMBs consist of several antibodies on their surfaces, one bead may bind more than one cell that can misinterpret the capture rate. Furthermore, most of the paramagnetic beads get stained with the cell marker. Therefore analyzing the supernatant fractions was a more sensible approach to measure the capture efficiency. However, also the bead fractions were analyzed by flow cytometer to identify bacterial contamination in the sample or to determine the sensitivity of the method, where live bacteria bound with bead show higher fluorescence compared to the unbound beads (Supplementary information: Figure S1). Therefore the method is applicable in the real life testing scenarios.

Carboxyfluorescein diacetate (CFDA) has been used to evaluate the viability of different cell types. This nonfluorescent hydrophobic compound diffuses through cell surface membranes into the cytoplasm. Upon hydrolysis by intracellular esterases, CFDA releases Carboxyfluorescein (CF), a green fluorescent product. Therefore green fluorescence indicates metabolic activity and membrane integrity of cells. However CF retention inside the cell depends on the cell type. Furthermore, CFDA is not able to enter into the cytoplasm of Gram-negative bacteria which consists of an additional outer membrane which consequently made it difficult to stain gram negative bacteria like Salmonella (Nikaido, 1996; Vaara, 1992).
To overcome this problem, the buffer was supplemented to increase permeability without destroying the viability. About $1 \mathrm{~mL}$ standard cell suspension of Salmonella containing $10^{6}$ cells $/ \mathrm{ml}$ suspended in different buffers was incubated with CFDA and detected in flow cytometry. Five different buffers were used for the experiments (see caption to Fig. S2) and the results show that HEPES (4-(2-hydroxyethyl)-1piperazineethanesulfonic acid) buffer $(50 \mathrm{mM}, \mathrm{pH} 8.0)$ containing $0.25 \%$ glutaraldehyde (GTA) provide the optimum condition for CFDA staining of gram-negative bacteria (Supplementary information: Figure S2). The main reason for the positive effect of the mentioned buffer is the cross linking nature of GTA that maximizes CFDA permeability into the cell and prevents CF expulsion out of the cell (Morono et al., 2004).

To further optimize the staining protocol a series of time periods $(1,5,10,20,30,45$ and $60 \mathrm{~min})$ for incubating bacteria and CFDA were studied. Ten minutes of incubation proved to be sufficient for a suitable discrimination of stained cells from control/unstained cells (Supplementary information: Figure S3). The results obtained from the experiment done with the optimized conditions and mentioned in Fig. 2 suggest that CFDA can be used as an indicator to detect viable bacteria after IMS.

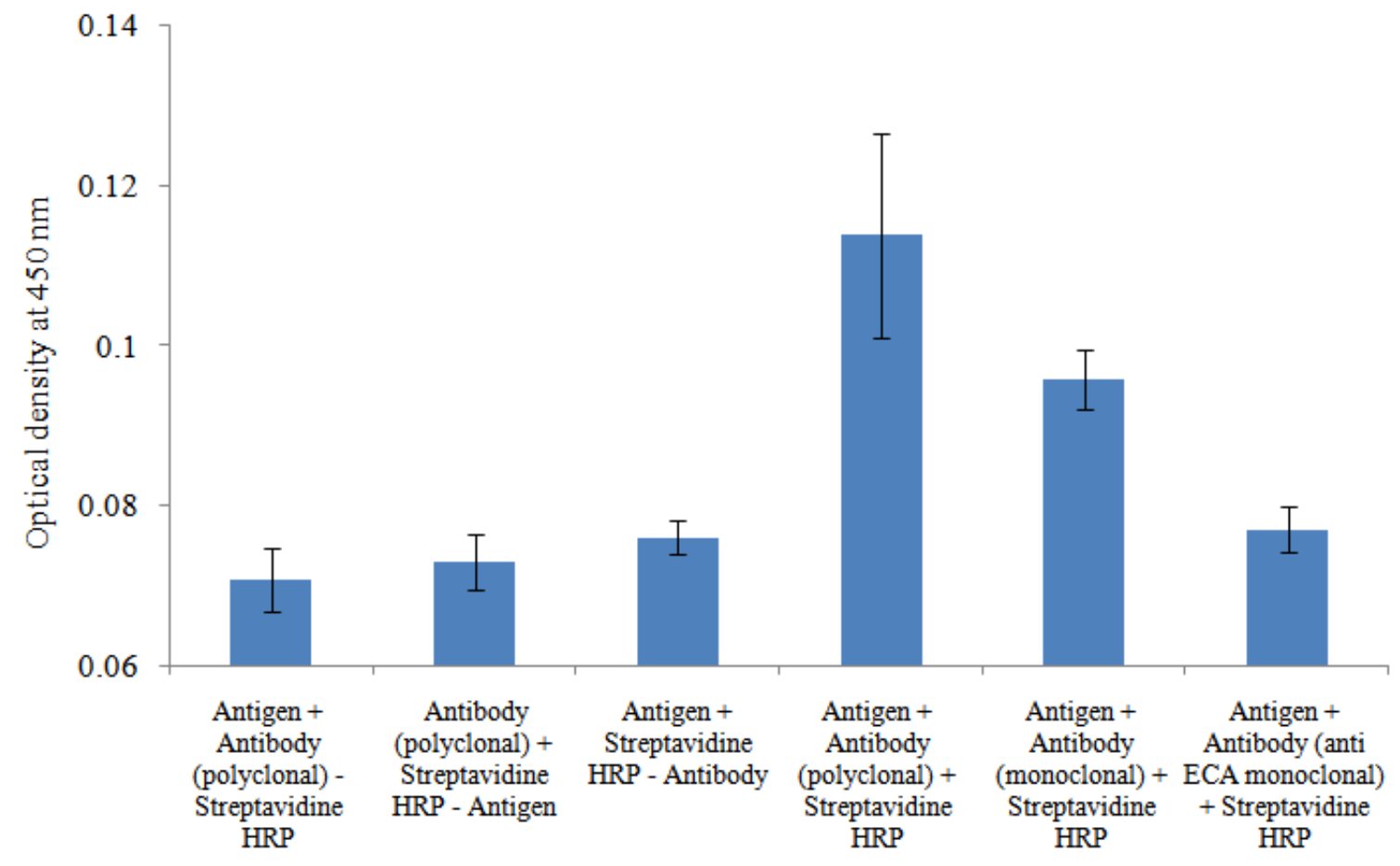

Fig. 1. ELISA reactivity of three different antibodies against Salmonella. Optical Density (OD) was measured at $450 \mathrm{~nm}$. The highest signal was observed from a Salmonella polyclonal antibody followed by Salmonella monoclonal antibody. The lowest activity was shown by ECA monoclonal antibody where the absorbance was similar to the controls (no antibody). The data is presented as the mean of three runs 


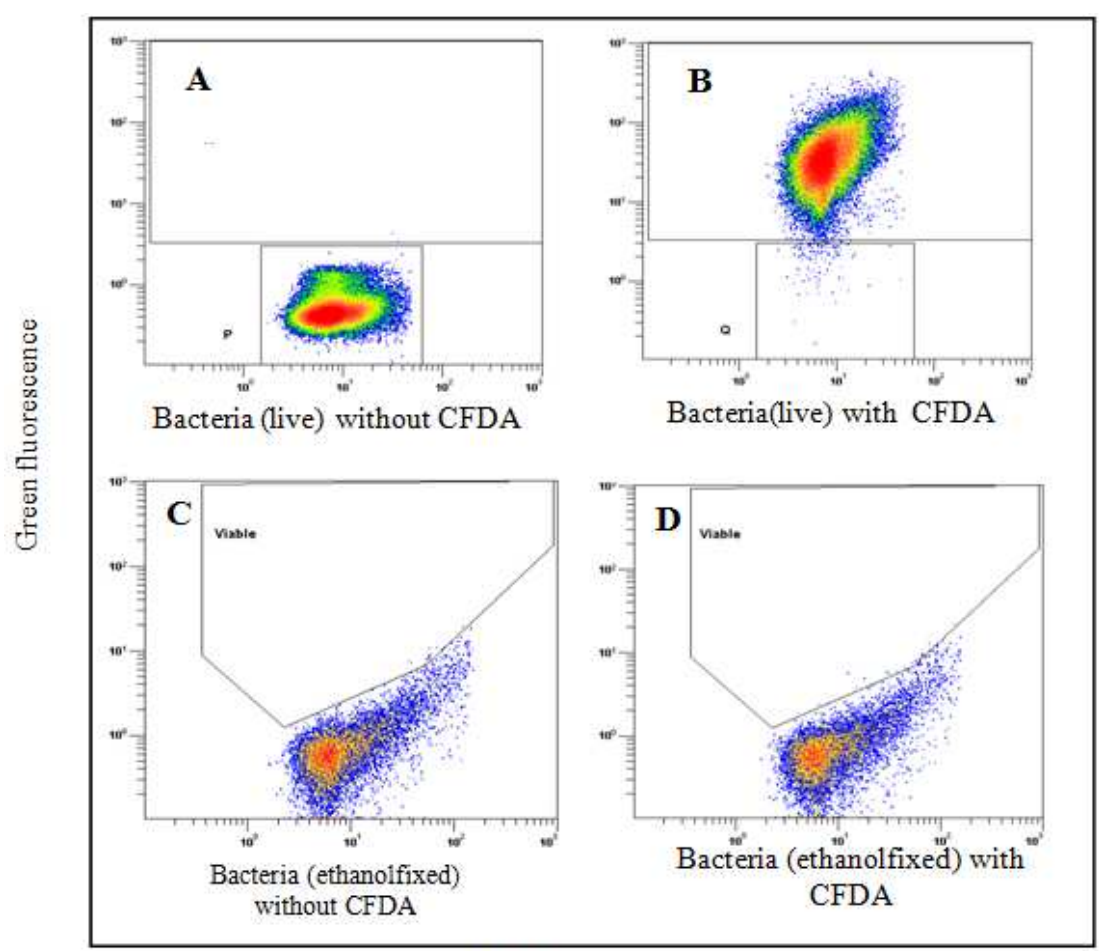

Forward scatter

Fig. 2. Dual-parameter histograms of viable $(A+B)$ and ethanol-fixed $(C+D)$ bacteria stained with and without CFDA and measured in flow cytometry. About $99 \%$ of viable bacteria show green fluorescence of carboxy- fluoresceine whereas almost $100 \%$ of ethanol-fixed bacteria were dead (CFDA negative)

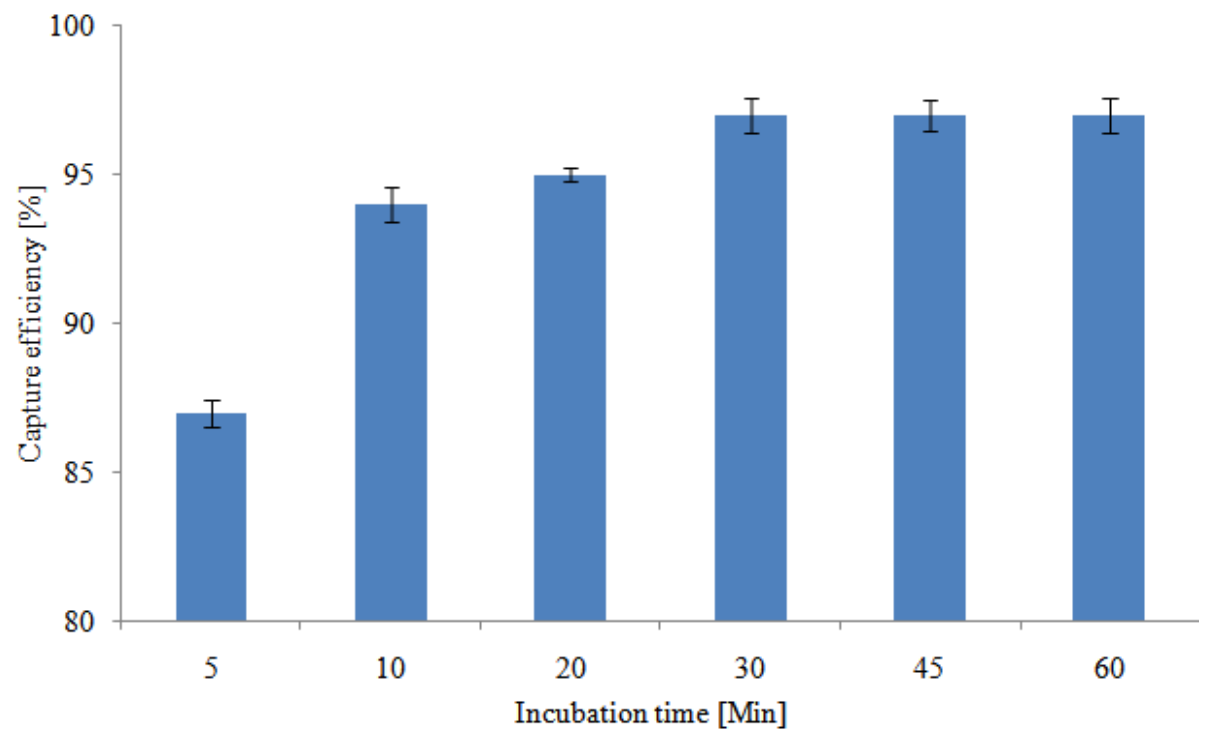

Fig. 3. Effect of incubation time on the capture efficiency for $10^{5}$ cells $/ \mathrm{mL}$ of Salmonella Typhimurium against $10^{8} \mathrm{IMBs} / \mathrm{mL}$ at room temperature. The data is presented as the average from three trials

\section{Determination of Suitable Incubation Time}

To investigate the effect of the incubation time of beads with bacteria on IMS efficiency, $1 \mathrm{~mL}$ portions of
Salmonella Typhimurium suspension containing $10^{5}$ bacteria were incubated with $10^{8}$ beads at room temperature for different time periods $(5,10,20,30,45$ and $60 \mathrm{~min}$ ) and the number of viable bacteria remaining 
after each incubation was analyzed by flow cytometry. Figure 3 shows that the capture efficiency increases from 87 to $97 \%$ with the incubation time increasing from 5 to $30 \mathrm{~min}$. Under the fixed concentrations of bead and bacteria the binding of cells to beads is a time-dependent process: The longer the incubation period, the higher the capture rate. However after a specific incubation period almost all cells have been captured. After that the captured cell number do not increase further even during the longest incubation period $(60 \mathrm{~min})$. As shown in the Fig. 3, it is sufficient to perform experiments with 30 min incubation time.

\section{Bead-Bacteria Interaction}

The capture efficiency can be altered by variation of the concentrations of paramagnetic beads and bacteria. To investigate the effect of bead concentration on IMS, several experiments were carried out using a fixed concentration of Salmonella Typhimurium with variable concentrations of IMBs. About $10^{5}$ to $10^{8}$ beads were added to each $1 \mathrm{~mL}$ Salmonella Typhimurium suspension containing $10^{5}$ cells, incubated for $30 \mathrm{~min}$ and then magnetically separated, finally measured by flow cytometry after subsequent CFDA staining for $10 \mathrm{~min}$. Figure 4 shows that the increasing bead concentration results in an increasing capture efficiency which rises sharply from 12 to 76 and then to $99 \%$ with every 10 fold increase in bead concentration. A minimum concentration of $10^{8} / \mathrm{ml}$ was required to separate $99 \%$ of $10^{5}$ bacteria where the ratio between bead and bacteria was $10^{3}$ to 1 .

\section{Influence of the Bacteria Concentration on IMS} Efficiency

Figure 5 shows the relationship between bacterial concentration and IMS efficiency. When the concentration of Salmonella was similar or lower than $10^{5} \mathrm{cells} / \mathrm{ml}$, the capture efficiency was about $98 \%$. The capture efficiency decreased from 98 to $75 \%$ and then to $20 \%$ with every 10 folds increase in Salmonella concentration. Optimized IMS conditions were applied for the experiments. The results demonstrate that the relationship between bead and bacteria concentrations is crucial for the IMS method. As already shown in Fig. 4, a large excess of beads compared to the bacterial cells was necessary to complete the immunoreaction and achieve $98 \%$ capture efficiency. This ratio decreases when we use higher number of bacteria and eventually increases the unbound cells.

\section{Specificity of IMBS}

For evaluating the specificity, IMBs containing rabbit polyclonal antibodies of Salmonella were used to capture Salmonella Typhimurium and three non-target bacteria; two strains of E. coli (K12 and MM294) and Listeria innocua. Both gram positive and negative bacteria were employed to check the effectiveness of our method. A fixed cell concentration $\left(10^{5}\right.$ bacteria $\left./ \mathrm{ml}\right)$ was used for all strains. As shown in Fig. 6, about 99\% Salmonella Typhimurium was captured by IMBs whereas the capture efficiency for non-specific strains was lower than $5 \%$.

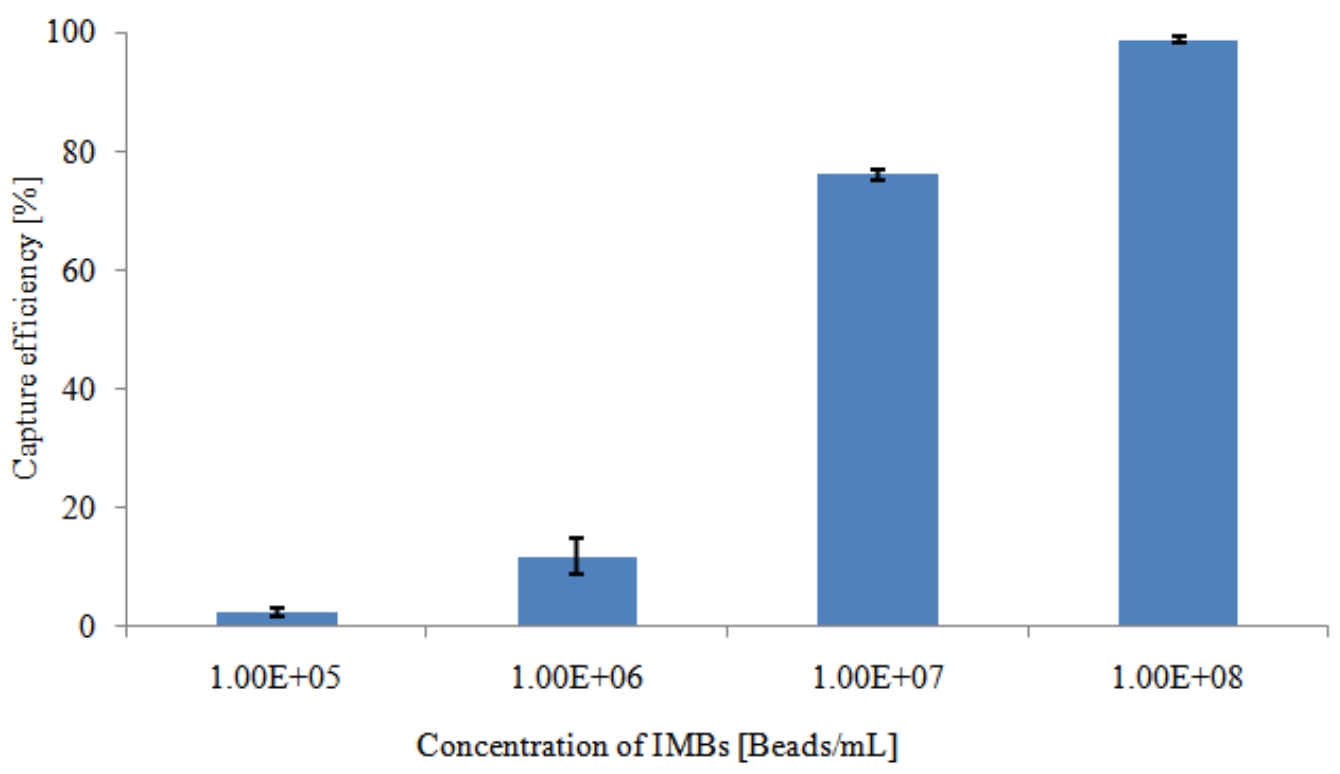

Fig. 4. Relationship between the proportion of separated cells and the bead concentrations in experiments with $10^{5}$ Salmonella Typhimurium $/ \mathrm{mL}$ and $1 \mu \mathrm{m}$ Dynabeads ${ }^{\circledR}$ (antibody coated) with an incubation period of $30 \mathrm{~min}$ at room temperature. The measurements are presented as the mean of three trials 


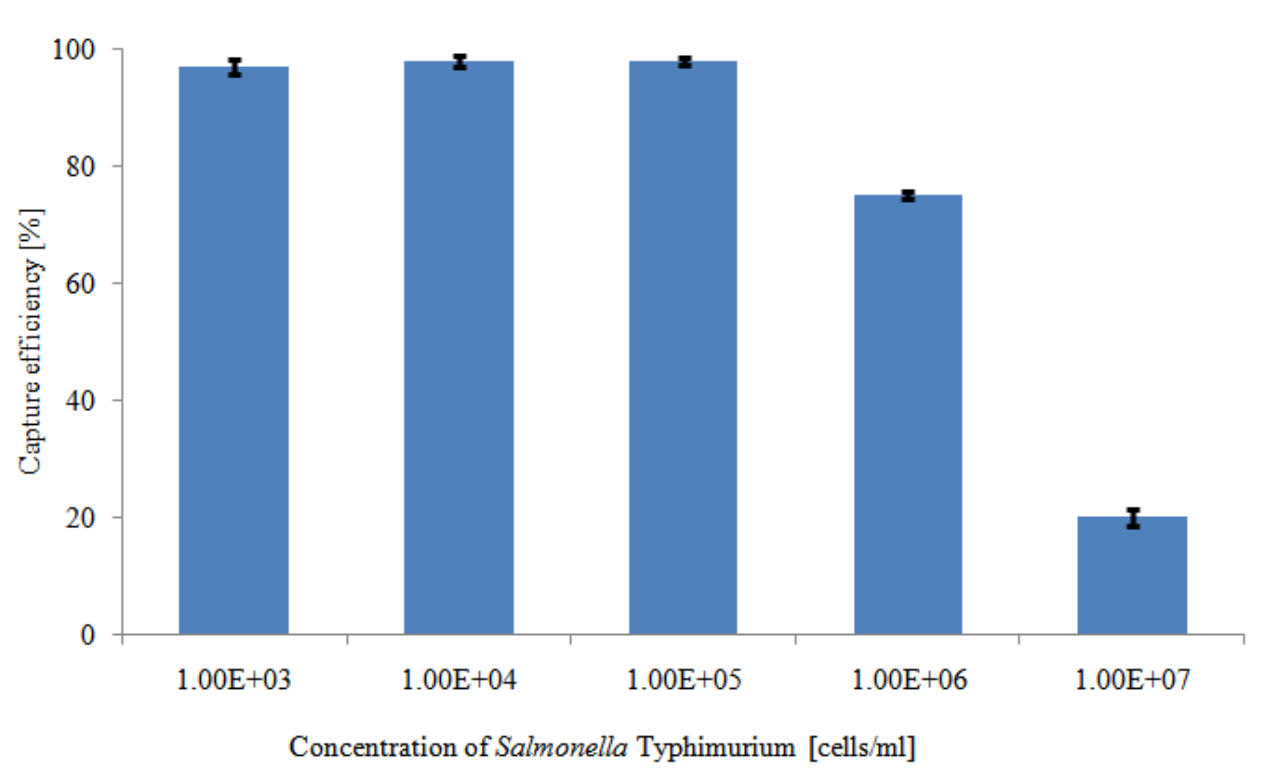

Fig. 5. Capture efficiency of $10^{8} \mathrm{IMBs} / \mathrm{mL}$ against the Salmonella Typhimurium concentration within the range $10^{3}-10^{7}$ cells $/ \mathrm{mL}$. The FCM assay conditions were the same as in Fig. 4. The data is presented as mean of three runs

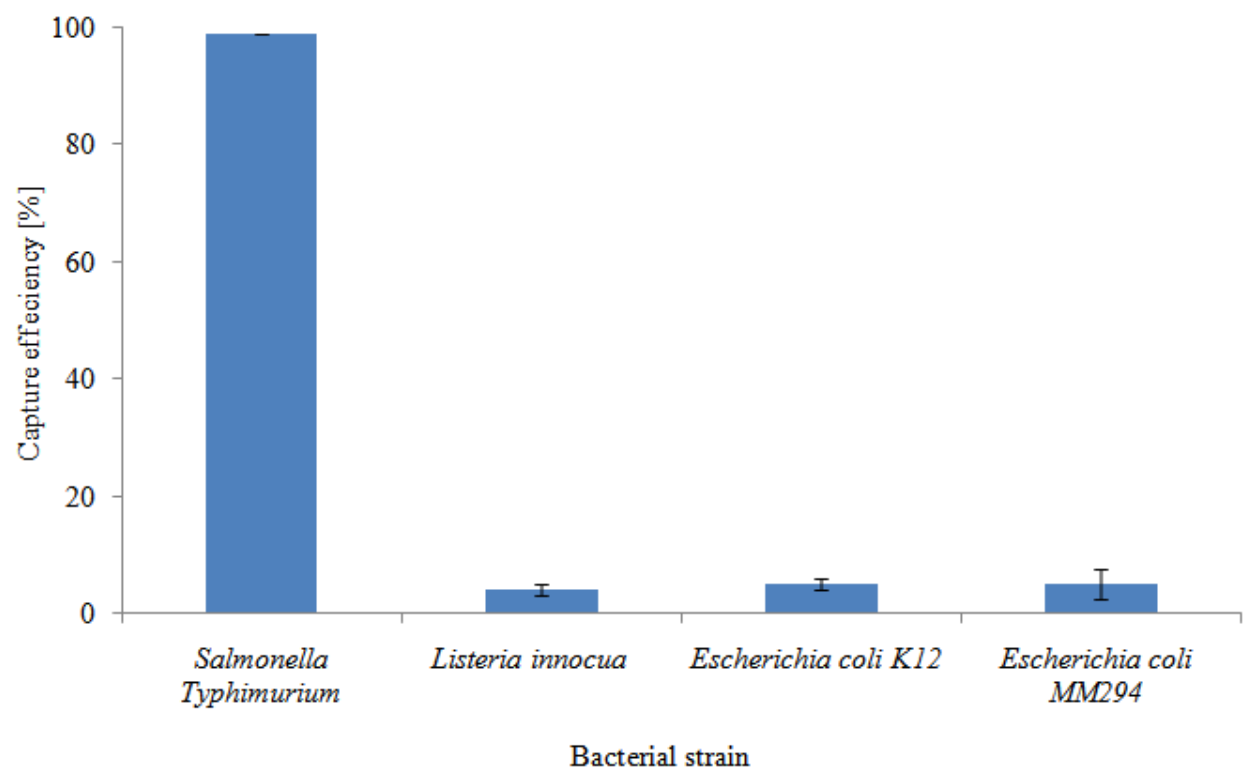

Fig. 6. The specificity of antibody to Salmonella Typhimurium and other species of bacteria, E. coli K12, E. coli MM294 and Listeria innocua. Each cell concentration was prepared to $10^{5} \mathrm{cell} / \mathrm{s} / \mathrm{mL}$. The FCM assay conditions were the same as in Fig. 4. The data is presented as mean of three runs

\section{Conclusion}

Immunomagnetic beads conjugated with polyclonal antibodies of Salmonella have been successfully applied to separate viable Salmonella Typhimuriumin in liquid samples, which are detected via flow cytometry. The major advantage of this method is that it allows for the enrichment and detection of viable bacteria. By coupling different antibodies to the magnetic beads, the choice of target cells can easily be changed. The parameters for IMS, e.g., bead and bacteria concentration, immunoreaction time and specificity have been optimized to improve capture efficiency. The developed method is suitable to obtain $98 \%$ capture efficiency from a wide range of bacterial concentrations. This holds significant potentials for the application onto an on-chip microfluidic environment. 


\section{Acknowledgement}

This project has been supported by a PhD grant from Ministry of Education and Science of SchleswigHolstein, Germany. We are thankful to Jan Lenke for his critical reading of the manuscript.

\section{Author's Contributions}

Shakil Ahmed: Designed the experiments, analyzed the data, prepared the manuscript, performed the experiments and read and approved the final manuscript.

Horst-Günter Rubahn and Helmut Erdmann: Designed the experiments, analyzed the data, prepared the manuscript, read and approved the final manuscript.

\section{Competing Interest}

The authors declare that they have no competing interests.

\section{References}

Batz, M.B., M.P. Doyle, J.G.Jr. Morris, J. Painter and R. Singh et al., 2005. Attributing illness to food. Emerg. Infect. Dis., 11: 993-999. PMID: 16022770

Bergquist, P.L., E.M. Hardiman, B.C. Ferrari and T. Winsley, 2009. Applications of flow cytometry in environmental microbiology and biotechnology. Extremophiles, 13: 389-401. PMID: 19301090

Cudjoe, K.S., T. Hagtvedt and R. Dainty, 1995. Immunomagnetic separation of Salmonella from foods and their detection using Immunomagnetic Particle (IMP)-ELISA. Int. J. Food Microbiol., 27: 11-25. PMID: 8527325

Fu, Z., S. Rogelj and T.L. Kieft, 2005. Rapid detection of Escherichia coli $\mathrm{O} 157: \mathrm{H} 7$ by immunomagnetic separation and real-time PCR. Int. J. Food Microbiol., 99: 47-57. PMID: 15718028

Füchslin, H.P., S. Kötzsch, H.A. Keserue and T. Egli, 2010. Rapid and quantitative detection of Legionella pneumophila applying immunomagnetic separation and flow cytometry. Cytometry A, 77: 264-274. PMID: 20099248

Hammes, F. and T. Egli, 2010. Cytometric methods for measuring bacteria in water: Advantages, pitfalls and applications.Analytical Bioanalytical Chem., 397: 1083-1095. PMID: 20352197

Hara-Kudo, Y., S. Kumagai, T. Masuda, K. Goto and K. Ohtsuka et al., 2001. Detection of Salmonella enteritidis in shell and liquid eggs using enrichment and plating. Int. J. Food Microbiol., 64: 395-399. PMID: 11294363

Hibi, K., A. Abe, E. Ohashi, K. Mitsubayashi and H. Ushio et al., 2006. Combination of immunomagnetic separation with flow cytometry for detection of Listeria monocytogenes. Analytica Chimica Acta, 573-574: 158-163. PMID: 17723519
Kunstmann-Olsen, C., 2012. Development of microfluidic sensing platforms for microbial contaminants and protocells. PhD Thesis, University of Southern Denmark.

McCabe-Sellers, B.J. and S.E. Beattie, 2004. Food safety: Emerging trends in foodborne illness surveillance and prevention. J. Am. Dietetic Assoc., 104: 1708-1717. PMID: 15499359

Mead, P.S., L. Slutsker, V. Dietz, L.F. McCaig and J.S. Bresee et al., 1999. Food-related illness and death in the United States. Emerg. Infect. Dis., 5: 607-625. PMID: 10511517

Morono, Y., S. Takano, K. Miyanaga, Y. Tanji and H. Unno et al., 2004. Application of glutaraldehyde for the staining of esterase-active cells with carboxyfluorescein diacetate. Biotechnol. Lett., 26: 379-383. PMID: 15104134

Nikaido, H., 1996. Outer Membrane. In: Escherichia coli and Salmonella: Cellular and Molecular Biology, Neidhardt, F.C., III. R. Curtiss, J.L. Ingraham, E.C.C., Low and B. Magasanik et al. (Eds), ASM Press, Washington, ISBN-10: 1555810845, pp: 29-47.

Seo, K.H., R.E. Brackett, J.F. Frank and S. Hilliard, 1998. Immunomagnetic separation and flow cytometry for rapid detection of Escherichia coli O157:H7. J. Food Prot., 61: 812-816. PMID: 9678161

USDA, 2008. Food Safety and Inspection Service. Microbiology Laboratory Guidebook Online, U.S. Department of Agriculture.

USFDA, 2002. Bacteriological Analytical Manual Online. Chapter 4a, U.S. Food and Drug Administration.

Vaara, M., 1992. Agents that increase the permeability of the outer membrane. Microbiol. Rev., 56: 395-411.

Vives-Rego, J., P. Lebaron and G. Nebe-von-Caron, 2000. Current and future applications of flow cytometry in aquatic microbiology. FEMS Microbiol. Rev., 24: 429-448. DOI: 10.1016/S0168-6445(00)00033-4

Wang, L., Y. Li and A. Mustaphai, 2007. Rapid and simultaneous quantitation of Escherichia coli 0157:H7, Salmonella and Shigella in ground beef by multiplex real-time PCR and immunomagnetic separation. J. Food Prot., 70: 1366-1372. PMID: 17612065

Yang, H., L. Qu, A.N. Wimbrow, J. Xiuping and Y. Sun, 2007. Rapid detection of Listeria monocytogenes by nanoparticle-based immunomagnetic separation and real-time PCR. Int. J. Food Microbiol., 118: 132138. PMID: 17716768 


\section{Supplementary Information}

1. Flow cytometry data showing contamination of bacteria (Salmonella Typhimurium) in liquid sample

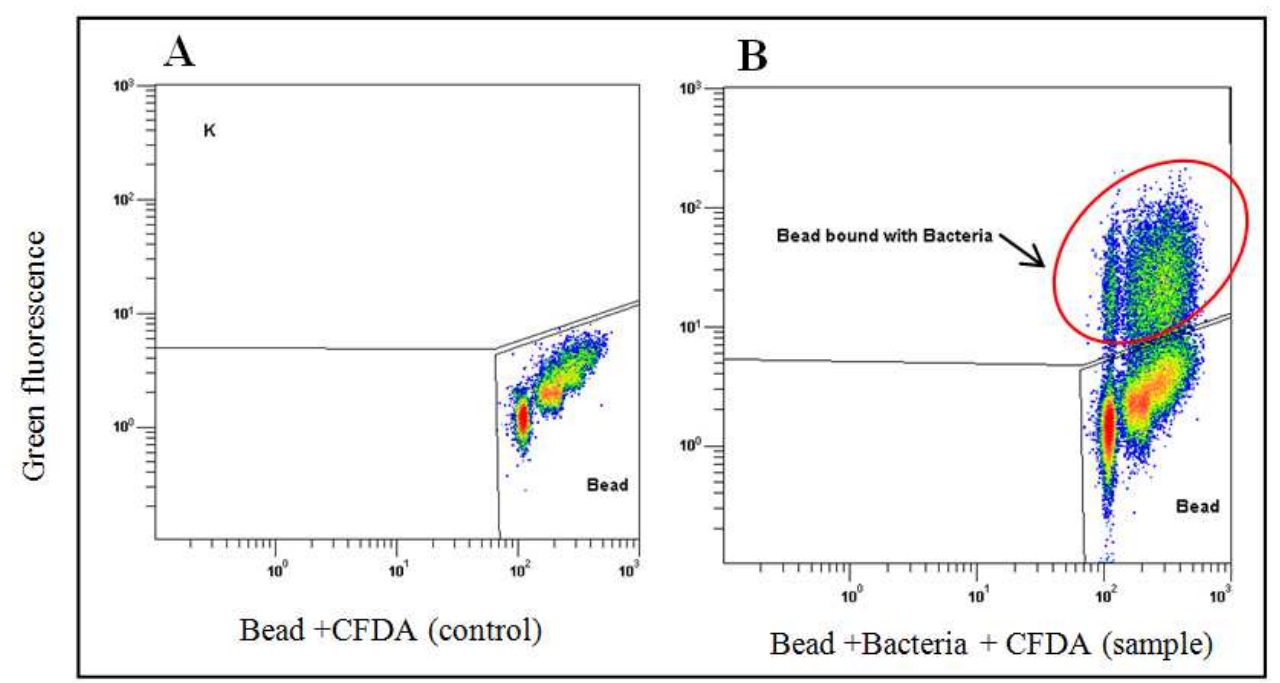

Forward scatter

Fig. S1. Flow cytometry results during conventional IMS. Bacterial contamination in the sample (B) can be easily distinguishable from control (A)

2. Image showing viability staining of Salmonella Typhimurium suspended in different buffers

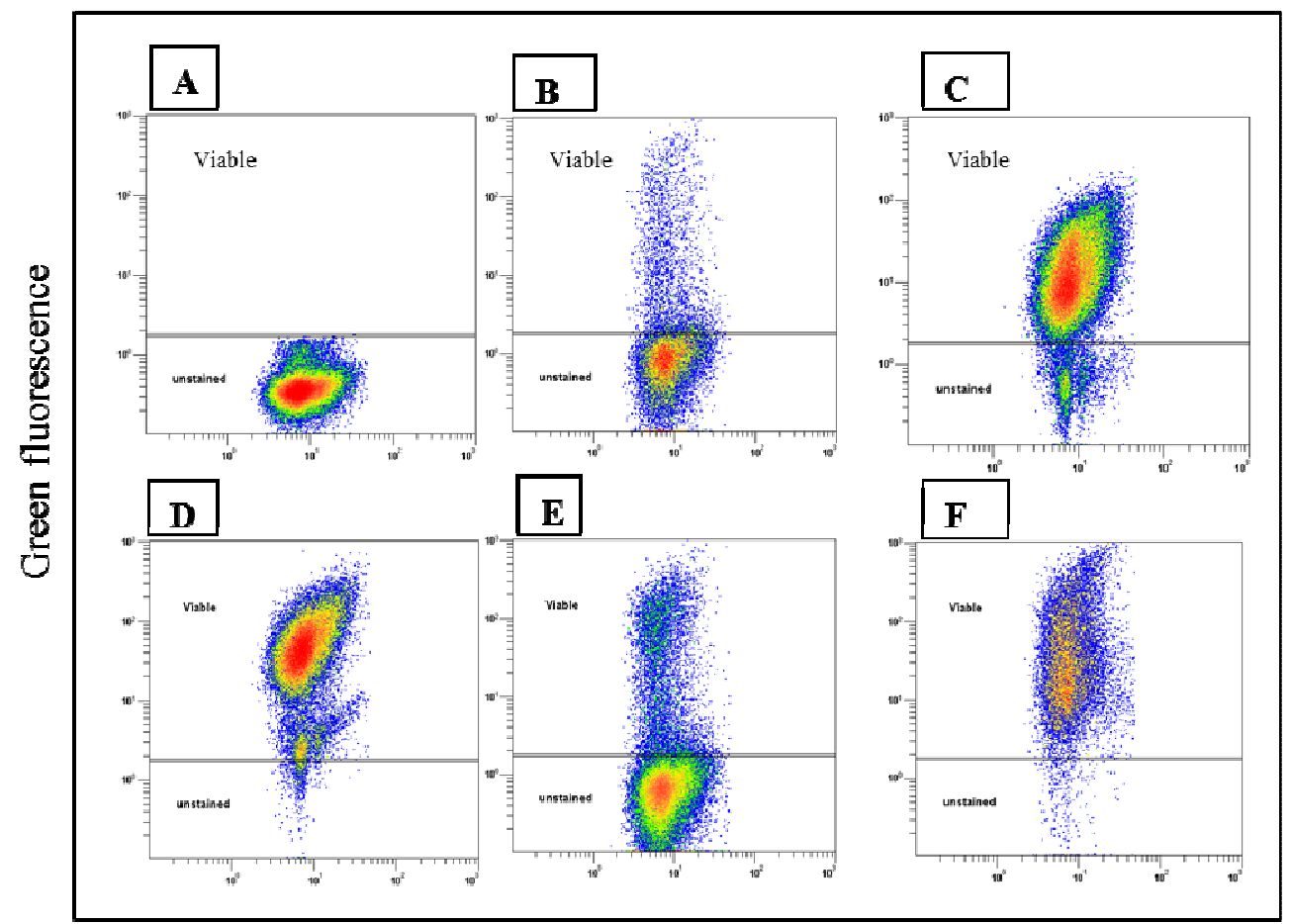

Forward Scatter

Fig. S2. Viability staining of Salmonella Typhimurium. Forward Scatter (FSC) Vs Fluorescence (FL1) plot showing the staining patterns of Salmonella in suspensions stained with CFDA at different buffers [A: unstained; B: PBS (pH 7.4) + Tween 0.01 \%; C:PBS (pH 7.0) + EDTA 0.5 mM + GTA 1\%; D:Hepes 50 mM (pH 8.0) + Glutaraldehyde 0.25 \%; E: Hepes 10 mM; F: Tris-Hcl $100 \mathrm{mM}+$ EDTA $1 \mathrm{mM}, \mathrm{pH} 8.0$ 
Gram-negative bacteria are highly impermeable to hydrophobic molecules. GTA has the ability to cross-link among the proteins in the outer membrane through reacting with amine groups (Reithmeier and Bragg, 1977). CFDA can enter into the cell because of the removal of hydrophilic amino groups as well as structural disorganization in the membrane. Because of cross linking of the peptidoglycan layer GTA prevents the leakage of CF from the cell (Hughes et al., 1970; Morono et al., 2004). The action of GTA on the bacterial cell membrane is believed to be the reason behind an improved efficiency of CFDA staining for gram-negative bacteria.

3. Graph showing the time course data of CFDA and bacteria (Salmonella Typhimurium) incubation

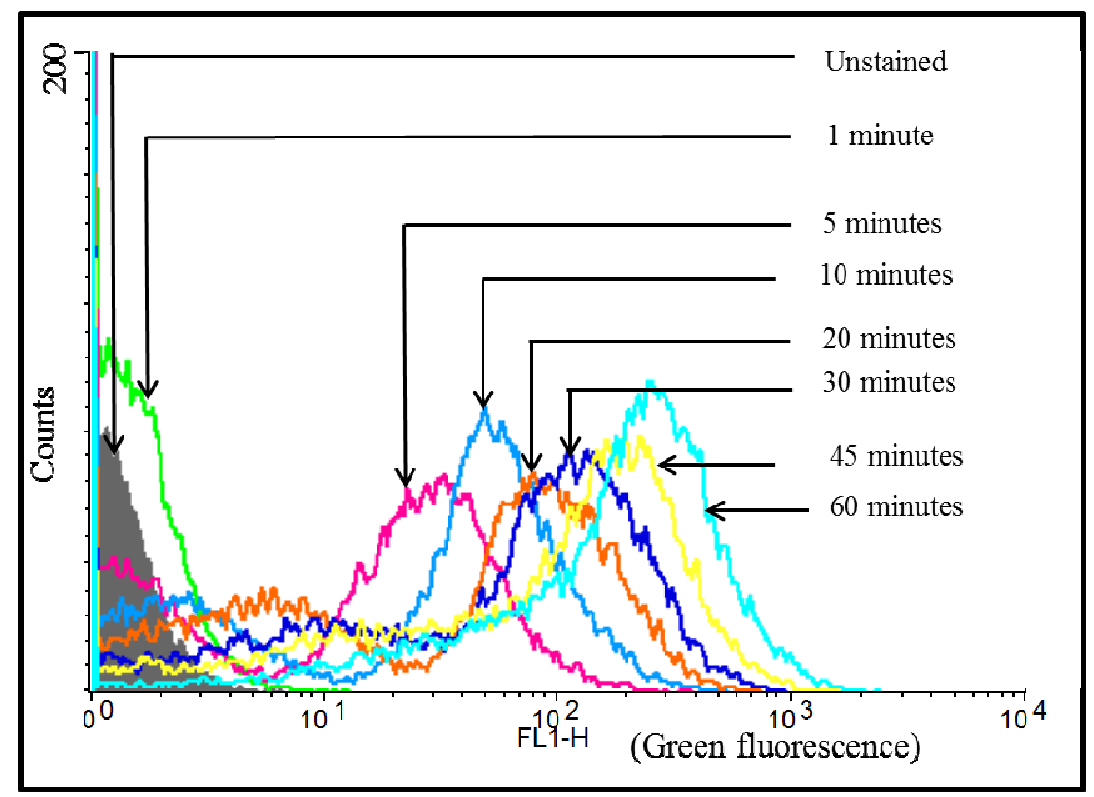

\section{References}

Hughes, R.C. and P.F. Thuman, 1970. Cross-linking of bacterial cell walls with glutaraldehyde. Biochem. J., 119: 925926. https://www.ncbi.nlm.nih.gov/pmc/articles/PMC1179494/

Morono, Y., S. Takano, K. Miyanaga, Y. Tanji and H. Unno et al., 2004. Application of glutaraldehyde for the staining of esterase-active cells with carboxyfluorescein diacetate. Biotechnol. Lett., 26: 379-383. PMID: 15104134

Reithmeier, R.A. and P.D. Bragg, 1977. Cross-linking of the proteins in the outer membrane of Escherichia coli. Biochim. Biophys. Acta, 466: 245-256. PMID: 322714 